\title{
An Architecture for aggregating real and virtual models in manufacturing systems
}

ASAMORI Motoo, KAMIO Youichi

Toyo Engineering Corporation

8-1, Akanehama 2-chome, Narashino-shi, Chiba 275, Japan asamori@is.toyo-eng.co.jp

MORI Kenichiro, YOSHIKAWA Norio, HOTTA Tadaaki OMRON Corporation 2-5-48, Tadao, Machida-shi, Tokyo 194, Japan

Kenichiro_Mori@omron.co.jp

KIMURA Toshiaki, GOTO Hiroyuki

Technical Research Institute, Japan Society for the Promotion of Machine Industry

1-1-12, Hachiman-cho, Higashikurume-shi, Tokyo 203, Japan kimura@tri.jspmi.or.jp

FUKUDA Yoshiro

Hosei University

3-7-2 Kagino-cho, Koganei-shi, Tokyo 184, Japan

fukuda@is.hosei.ac.jp

\begin{abstract}
A manufacturing system needs to adapt or renew itself rapidly in order to produce better and cheaper products that customers require. In this paper a new architecture is proposed that features "renewal" within the life cycle of production
\end{abstract}


system. In order to support renewal effectively the architecture includes not only the feed-forward structure of plan, design, construction, operation, and maintenance, but also feedback information about know-how and experiences of field operations. The architecture assumes a virtual enterprise environment which accounts for and unifies both design and manufacturing even though they typically operate remotely. The architecture supports gathering and categorizing multimedia data such as signals from equipment, trouble information, quality management information, operators' know-how, etc. A demonstration, based on actual field-gathered multimedia data and its associated renewal activities, will verify the feasibility of the renewal-centered architecture. The demonstration will use object-oriented models and simulators.

\section{Keywords}

Renewal, Virtual Manufacturing, Real Model, Virtual Model, KAIZEN Activity

\section{INTRODUCTION}

The environment surrounding the manufacturing industries is changing substantially. These changes include structural changes in supply and demand, development and applications of new technology, and various new measures for environmental conservation. Under these changes, the life of manufacturing systems tends to become relatively longer compared with that of product. As the industries policy for manufacturing system changes from scrap and build to improvement, renewal activity in production life cycle is recognized more important than ever.

Although facility life cycle and virtual manufacturing system are studied, the industries are not sufficiently equipped with tools, evaluation methodology and technology that should systematically support all phases from planning, designing, construction, operation, maintenance to disposal of manufacturing devices. As production activities of enterprises become more international, it is often observed that a manufacturing system is operated remotely from the designing department. In such a case, remote operation and virtual enterprise are studied to ensure smooth information exchange between departments and to modify or change the system efficiently and at a low cost in accordance with changes in manufacturing environment. Advances in information technology proceed rapidly, and new tools and technology are introduced in market to apply to every phase of the life cycle of manufacturing system.

In view of these trends, a new platform was proposed which aggregates real and virtual models without the consciousness of geographical distance, and can compose, operate and evaluate a manufacturing system of shop floor level in design, operation, maintenance, and improvement phases. Through experimental evaluation, we verified that the platform is feasible and can efficiently realize the 
improvement of productivity of manufacturing systems. On the basis of this result, a new reference architecture focused on renewal activity of manufacturing system is proposed, which consists of a function model and an implementation model. Modeling discussed in this paper is limited to manufacturing systems which include devices, operators, materials, and information processed in the shop floor level.

In this paper, Chapter 2 defines renewal activity which we focus on. Chapter 3 describes the definition of real and virtual models, and the need for aggregation of them. Chapter 4 introduces the requirements for renewal activity, and Chapter 5 proposes a new reference architecture of renewal activity.

This study has been made as "the Globeman 21 project", a part of IMS International R\&D Project.

\section{KAIZEN ACTIVITY AND RENEWAL ACTIVITY}

Nowadays the product life is shorter than ever. The production system adopts or renews itself in accordance with the change of product. Some of module units, which compose manufacturing equipment, are replaced frequently. It is important to keep the same or better performance by continuously monitoring and maintaining the facilities in normal condition in the operation phase as defined in the design phase even though the system configuration of the manufacturing facilities is changed. In many Japanese companies, the manufacturing system is kept well through continuous KAIZEN activity. KAIZEN activity is based on the local shop floor level, and has been carried out in a small group of several operators and maintenance persons. Therefore, KAIZEN activity can not always be applied to the global manufacturing environment, because it is not fully supported by the information system technology.

This research is intended to realize the environment which supports KAIZEN activity using up-to-date information technology. It is supposed that the model of manufacturing system is built in the design phase and that the information of it is kept in the electronic data. The operating information is monitored from the running manufacturing facilities. If any difference between the model information and the monitored data is observed, the monitored data is transferred to the model. Through the simulation carried out using the monitored data, the cause of the difference is investigated and counter measure is considered and decided for implementation. The manufacturing system is re-configured to cope with the situation (major change). This activity is carried out in the environment which has already been proposed in our R\&D activity. In case there is no need of reconfiguration, another solution is taken so that some parameters are changed to meet the situation (minor change). These activities are called "RENEWAL " as shown in figure 1. 


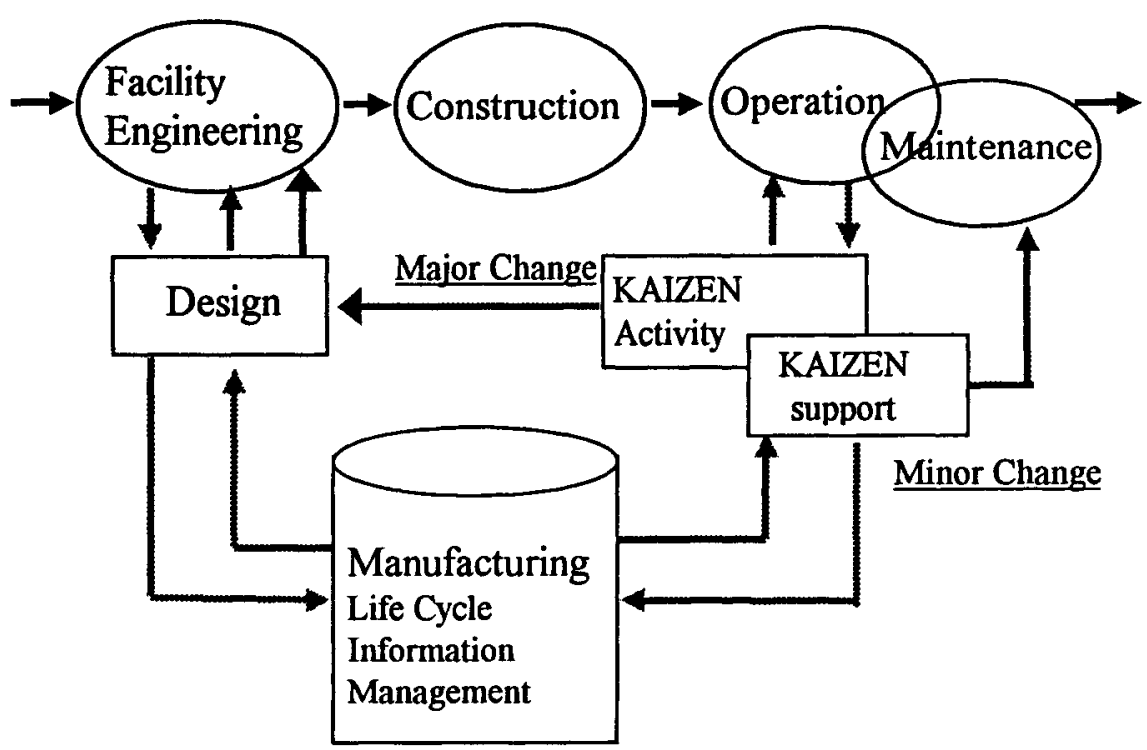

Figure 1 Life Cycle of Manufacturing System and Renewals.

\section{AGGREGATION OF REAL AND VIRTUAL MODELS}

An actually existing manufacturing system is composed of devices, control information, management information, material flow, and transaction processes among them. Design, implementation and operation of a manufacturing system are very complicated because all the elements above are handled together, and therefore problems are difficult to identify if they occur. In order to identify a problem, a specific viewpoint must be established, and the model is built from that point which is selected from many viewpoints such as productivity of manufacturing system, operability of devices, quality of products, etc. relating to the problem.

A model represents the behavior of manufacturing system on several types of simulators or in other information processing world. It is called a virtual model. The virtual model in design phase is made, tested and evaluated from a critical point of view in manufacturing system. Many case studies are carried out using the model until the model is fixed to meet the requirements. A virtual model expresses the ideal states and their transition of manufacturing facilities under given conditions in the virtual world. 
The virtual model of a manufacturing system has been built and used as a part of designing tools, and it is considered sufficient only in the workflow from designing, implementation to operation of manufacturing system. But taking account of renewal activity, the model needs to represent an existing manufacturing system in the real world. It is called a real model which is made up of the monitored and measured data of running devices from the viewpoint of specific information. The behavior of a manufacturing system in the real world can be described using the real data.

The virtual model is made and used mainly in design phase, while the real model is in operation phase. In renewal activity, a new environment is required where the real and virtual models are aggregated as mentioned in Chapter 2 "Kaizen Activity and Renewal Activity." The manufacturing system is evaluated more efficiently and effectively.

\section{REQUIREMENTS FOR RENEWAL ACTIVITY}

A manufacturing system consists of various devices which have their own information items and structures. Information of a device is independently defined and is not compatible with that of others, and it is difficult to exchange information among devices. To realize information exchange, an interface program is needed, which is not general but particular. Therefore a template is needed which shows the generic meaning of information independent of the structure dedicated to the device. There is too much information to be treated in a lump, and it is difficult to find out the necessary information. To simplify the problem, the template should be individually built in the specific point of view, however it should be furnished with common information items and structure.

The information of these models tends to be exchanged at different places in global manufacturing environment rather than in the same factory. The communication function is required in global manufacturing environment independent of the spatial distance.

Renewal activity is intended to be the same activity as Japanese KAIZEN activity. So the reference model deals with not only on-off signal information of the manufacturing devices but also human oriented information such as audio and visual signals treated by operators and maintenance persons. In addition to the character and numerical data processed on the computer, multimedia information such as picture, image, voice, animation, etc. is required to be treated in integrated and inter-related forms for the purpose of renewal activity. 


\section{REFERENCE ARCHITECTURE}

The importance of aggregating the real and virtual models in renewal activity is described in Chapter 3 " Aggregation of Real Model and Virtual Model" in this paper. In order to realize that, two new reference architectures are proposed, one for building models and the other for implementing models.

\subsection{Reference architecture for building models}

The necessity of template in building models is described in Chapter 4 "Requirements for Renewal Activity" in this paper. A new reference architecture is shown in Figure 2 as a conceptual platform for building models of a manufacturing system.

The template is necessary for smooth exchange of information not only between real models in manufacturing system but also between real and virtual models. The template is an interface model of information exchange. It is not a functional model, but includes a functional aspect. The template is created from various points of views, such as productivity, manageability, operability, maintainability, quality assurance and so on, in manufacturing system. It describes briefly the meanings of information in order not to depend on the information items and its structure dedicated to manufacturing devices. It is common to all communication interfaces of real and virtual models, and is provided as a library based on objectoriented methodology. The instance of real and virtual models is created using this class library from the specific point of view.

\subsection{Reference architecture for implementing models}

The other reference architecture is proposed as shown in Figure 3 as a conceptual platform for implementation of manufacturing system.

This architecture consists of the following four layers:

Layer 1 : Common Platform - Represents communication network of data exchanges.

Layer 2 : Object Models - Represents information models of real and virtual manufacturing systems with communication interfaces through which data is transferred to the communication network, including multimedia database.

Layer 3 : Tools - Represents manufacturing equipment and devices including its controller such as NC and robot, data gathering devices like POP (point of production), and simulation tools for design engineering.

Layer 4 : Users - Represents designer, operator and manager who use the information of manufacturing system. 
This architecture provides solutions for the requirements mentioned in Chapter 4 "Requirements for Renewal Activity."

The information exchange between the instances of real and virtual is carried out through Layer 1 (Common Platform). The communication in remote manufacturing environment is realized through Layer 1 and Layer 2 (Object Models). Communication network is realized based on information technology of Internet and LAN (Local Area Network) in the factory, etc., and interfaces are built using common off-the-shelf tools such as JAVA, C++, and so on. This is an infrastructure for data exchange between engineering department and manufacturing one. It removes the restrictions of time and space, and leads to promotion of smooth business activities in the global manufacturing environment.

There are many useful data in the real manufacturing system, and operators have knowledge and know-how about it. Such information leads to needs and seeds for renewal activity of manufacturing system. But it is buried in the files of field operation reports or in the head of operator. Two steps are needed for the utilization of information in the field, one is to get information, and the other is to store it and retrieve necessary pieces. The former is realized in layer 3 (Tools), where multimedia devices, e.g. video camera and voice recorder, are used for gathering information from operators in the real manufacturing field. The latter belongs to database in layer 2. Database archives raw data as they were. In renewal activity field data are analyzed from various points of view, and they leads to creation of new idea for improving the manufacturing system.

Object model is built using the template, and has an interface structure common for communication with other object models. In renewal activity, there are two cases of aggregation of real and virtual models. One is that real model is treated in the same manner as the virtual model in the virtual world. The other is that virtual data is replaced or incorporated with real data in the virtual model. These aggregations are realized in layers $2 \& 3$ of the virtual world.

Tools stand for data gathering and displaying devices through which an operator monitors the status of manufacturing facilities and inputs control/management information in real world. They express simulator and calculation software in virtual world. This layer includes multimedia equipment and graphical user interface for supporting virtual reality.

Users mean operators and field managers in the factory, and engineers and designers in product and process engineering. They have knowledge and knowhow, and they decide what and how to perform renewal activity. 


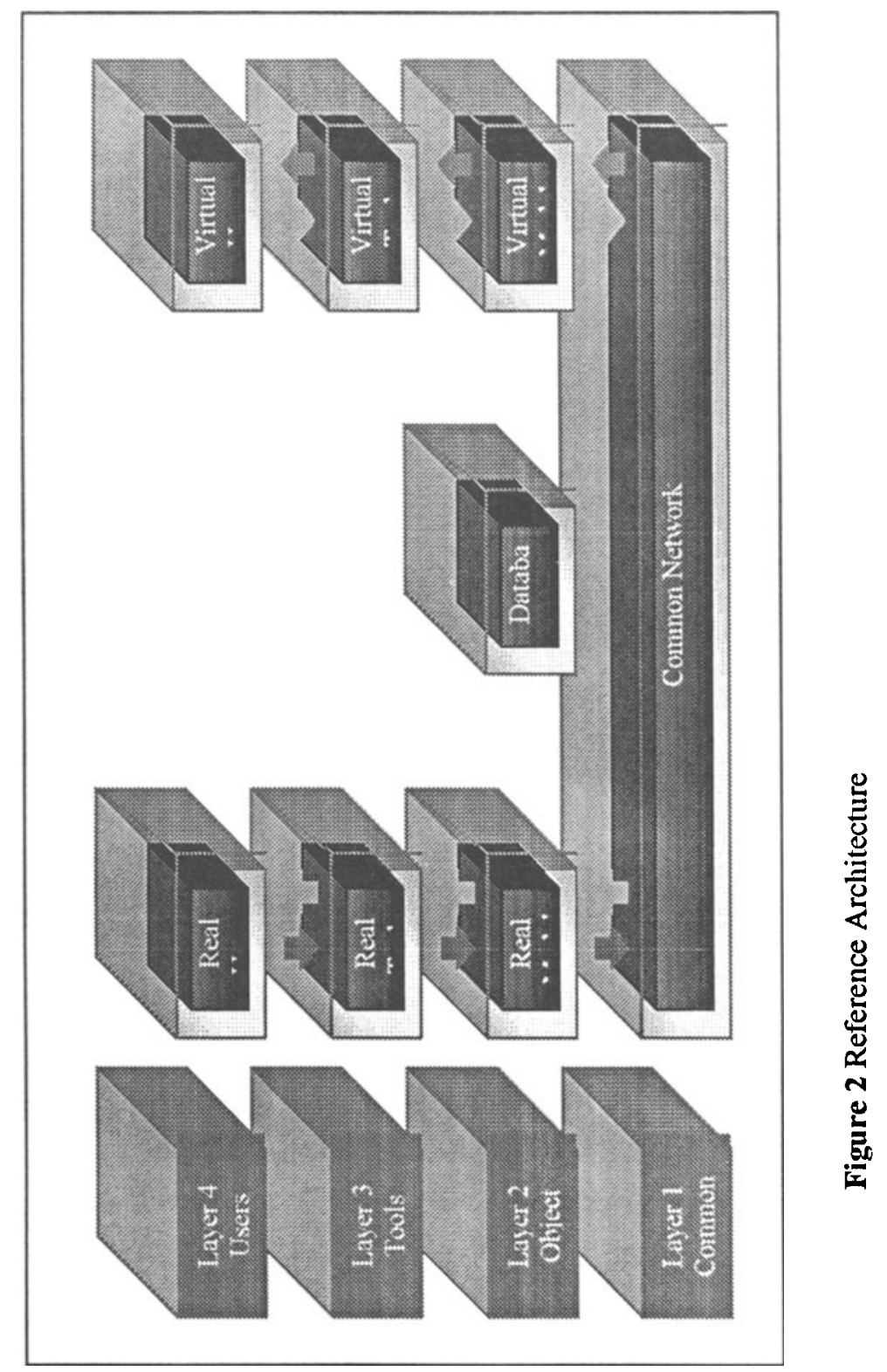




\section{CONCLUDING REMARKS}

Two new reference architectures are proposed in focus on renewal activity in the life cycle of manufacturing system. A demonstration system is prepared for validation of the reference architectures. A sample manufacturing system is selected as a candidate for demonstration, and information of its features and specifications are gathered from designing section and operation field in the factory.

We are now under designing, and in near future we will build a demonstration system and will report the result.

\section{REFERENCES}

Iwata, K. et al. (1995). Proceedings of EI '95, pp.154-167.

Onosato, M. and Iwata, K. (1993). Annals of the CIRP Vol.42, Nol, pp.475-478, CIRP

Mitsuishi, M. et al. (1995). Proceedings of IROS' 95, pp. 13-20, IEEE

NIIIP. (1995). NIIIP cycle0, Revision 6, The National Industrial Information Infrastructure Protocols Consortium.

IMS Promotion Center (1996). Enterprise Integration for Global Manufacturing Towards $21^{\text {st }}$ Century

Mori, K. et al. (1996). Proceedings of APMS'96, pp.309-314, IFIP (http://ims.toyo-eng.co.jp/pub/gm2l pub/PUBLICATION/apms/apms.htm) 